This is a self-archived version of an original article.

This reprint may differ from the original in pagination and typographic detail.

Title: Effects of Thermal Neutron Irradiation on a Self-Refresh DRAM

Author(s): Lucas Matana Luza, Daniel Söderström, Helmut Puchner, Rubén García Alía, Manon Letiche, Alberto Bosio and Luigi Dilillo

DOI: $10.1109 / D T I S 48698.2020 .9080918$

Published: 30 April 2020

Document version: Post-print version (Final draft)

Please cite the original version:

L. M. Luza et al., "Effects of Thermal Neutron Irradiation on a Self-Refresh DRAM," 2020 15th Design \&

Technology of Integrated Systems in Nanoscale Era (DTIS), 2020, pp. 1-6, doi: 10.1109/DTIS48698.2020.9080918.

This material is protected by copyright and other intellectual property rights, and duplication or sale of all or part of any of the repository collections is not permitted, except that material may be duplicated by you for your research use or educational purposes in electronic or print form. You must obtain permission for any other use. Electronic or print copies may not be offered, whether for sale or otherwise to anyone who is not an authorised user. 


\title{
Effects of Thermal Neutron Irradiation on a Self-Refresh DRAM
}

\author{
Lucas Matana Luza ${ }^{1}$, Daniel Söderström², Helmut Puchner ${ }^{3}$, Rubén García Alía ${ }^{4}$, Manon Letiche ${ }^{5}$, \\ Alberto Bosio ${ }^{6}$ and Luigi Dilillo ${ }^{1}$ \\ ${ }^{1}$ LIRMM, University of Montpellier, Montpellier, France, * $\{$ lucas.matana-luza, dilillo\}@lirmm.fr \\ 2 Department of Physics, University of Jyväskylä, Jyväskylä, Finland, *daniel.p.soderstrom@jyu.fi \\ ${ }^{3}$ Cypress Semiconductor, San Jose, USA, *helmut.puchner@cypress.com \\ ${ }^{4}$ Engineering Department, CERN, Geneva, Switzerland, *ruben.garcia.alia@cern.ch \\ 5 Institut Laue-Langevin, Grenoble, France, *letiche@ill.fr \\ ${ }^{6}$ Lyon Institute of Nanotechnology, École Centrale de Lyon, France, *alberto.bosio@ec-lyon.fr
}

\begin{abstract}
In this study, static and dynamic test methods were used to define the response of a self-refresh DRAM under thermal neutron irradiation. The neutron-induced failures were investigated and characterized by event cross-sections, soft-error rate and bitmaps evaluations, leading to an identification of permanent and temporarily stuck cells, block errors, and single-bit upsets.
\end{abstract}

\section{Index Terms}

neutron, irradiation, Self-Refresh, DRAM, SEE, HyperRAM

\section{INTRODUCTION}

Thermal neutrons are generated by reducing the kinetic energy of energetic neutrons through a moderator, reaching an average energy of $25 \mathrm{meV}$ at room temperature. The reaction of thermal neutrons with the boron-10 $\left({ }^{10} \mathrm{~B}\right)$ generates byproducts (including an alpha particle and a lithium-7 nucleus) that can cause single event upsets (SEUs). These effects were a concern for static random access memories (SRAMs) and dynamic random access memories (DRAMs) fabricated with borophosphosilicate glass (BSPG) during the 90s. Nowadays, the BPSG is not present in these devices [1]-[4].

However, several works have been done in sub-micron SRAM devices, which show that even without the BSPG layer in advanced $\mathrm{Si}$ technologies, there is a high possibility of contamination during the fabrication process [5]-[9], and that the impact of thermal neutrons should not be ignored [10]-[12]. In [7], the authors investigate the thermal neutron sensitivity of a $40 \mathrm{~nm}$ SRAM in which they present a residual source of ${ }^{10} \mathrm{~B}$ from doping in silicon. A characterization of SEUs in Xilinx $20 \mathrm{~nm}$ UltraScale Kintex FPGA is presented in [13], resulting in a cross-section comparison on devices with and without a technique to mitigate the effects under thermal neutron irradiation. Also, as presented in [14], the contribution of thermal-neutron-induced soft-error rate (SER) in 16-nm bulk FinFET flip-flops can be comparable with the high-energy-neutron-induced SER, showing variations for different flip-flops designs, owing to different ${ }^{10} \mathrm{~B}$ contamination and different critical charge values.

The proposed work represents the first study on the effects of thermal neutron irradiation on a self-refresh DRAM, a novel type of memory device. The rest of the paper is structured as follows: Section II presents the Device Under Test (DUT), the test facility and the experimental setup; Section III describes the applied test modes; Section IV presents and analyzes the results from the thermal neutron irradiation; Section $\mathrm{V}$ concludes the work.

\section{TEst SETup}

\section{A. Device Under Test}

The DUT is the S27KS0642GABHI020, a 64 Mib HyperRAM $^{\text {TM }}$ Self-Refresh DRAM manufactured by Cypress Semiconductor. The DUT is a high-speed CMOS with a HyperBus ${ }^{T M}$ interface, which uses the Double Data Rate (DDR) to reach a data throughput up to $400 \mathrm{MBps}$ with a maximum clock rate of $200 \mathrm{MHz}$. The memory is laid out

This study has been achieved thanks to the financial support of the VAN ALLEN Foundation (Contract No. UM 181387) and the Region Occitanie (Contract No. UM 181386)

This study has received funding from the European Union's Horizon 2020 research and innovation programme under the MSC grant agreement no. 721624 .

The experiment(s) on D50 (INDU-178) at ILL have been performed within the "Characterisation Program" of the IRT Nanoelec, co-funded by the French government in the frame of the "Programme d'Investissements d'Avenir" under the reference ANR-10- AIRT-05. 
on a $38 \mathrm{~nm}$ technology, and the cells array is composed of 8192 rows, and each row contains 512 word (16 bits) address.

\section{B. Test Facility}

The tests were carried out at the Platform for Advanced Characterisation (PAC-G) facility that is hosted by the Institute Laue Langevin (ILL) in Grenoble, France, using the D50 instrument. This instrument provides thermal neutrons moderated by liquid deuterium at $20 \mathrm{~K}$, and the captured flux (i.e., equivalent flux of 25 meV neutrons) is adjustable from 0 to $10^{10}$ particles $/ \mathrm{cm}^{2} / \mathrm{s}$, which is controlled by a ${ }^{3} \mathrm{He}$-detector and periodical gold foil measurements [15]. Fig. 1 presents the energy and wavelength beam spectrum provided by the facility.

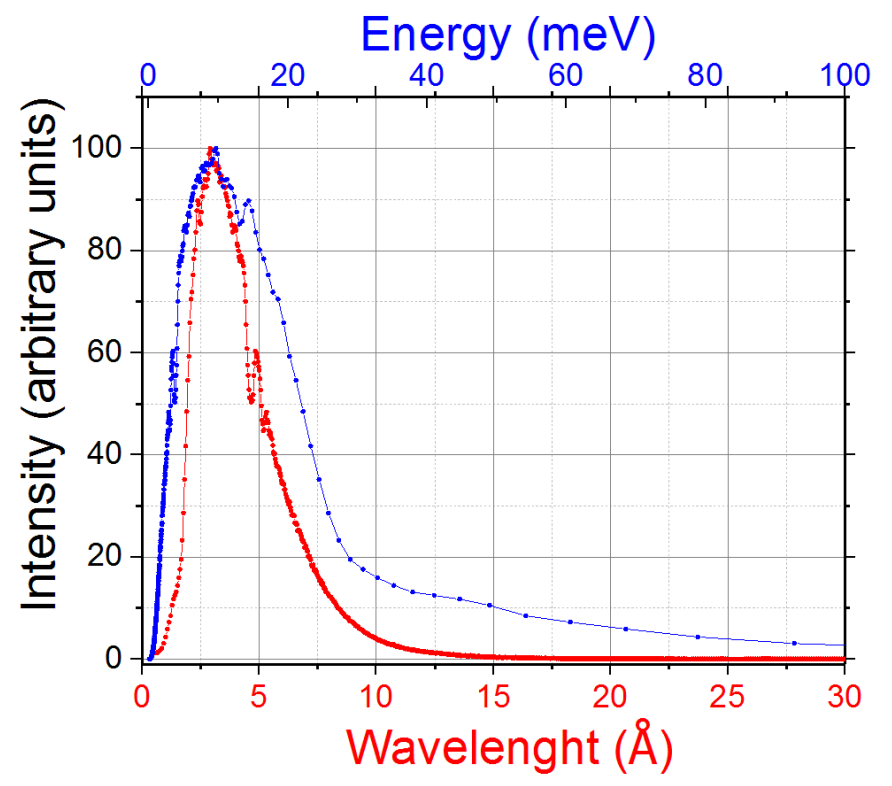

Fig. 1. Energy and wavelength beam profile.

\section{Test Setup}

The test setup is composed of a control board based on the Zynq-7000 SoC from Xilinx and a daughter board carrying the DUT. Fig. 2 presents both these boards. The controller system uses the System-on-Chip (SoC) ARM Cortex $^{\text {TM }}$-A9 processor to perform the test algorithms on the DUT through the HyperBus ${ }^{\text {TM }}$ controller, which is an IP (Intellectual Property) provided by Cypress and implemented in the SoC's Programmable Logic, which manages the communication between the processor and the DUT.

During the tests, the power supply was monitored in order to identify single event latch-up (SEL). All performed tests were logged with the logical address, bit error data, and operation status. Functional tests were performed between the runs to ensure the full functionality of the device. The DUT was tested under room temperature and nominal supply voltage, using a $25 \mathrm{meV}$ thermal neutron equivalent flux of $10^{9}$ particles $/ \mathrm{cm}^{2} / \mathrm{s}$ with a $30 \times 30 \mathrm{~mm}^{2}$ beam. The control board was positioned out of the beam, and to ensure the system reliability, the same was also shielded using a boron carbide material [16].

\section{TEST MODES}

In this study, to evaluate the memory response during irradiation, static, and dynamic memory tests were applied to the DUT. Dynamic tests constantly access the memory employing read and write operations in order to emulate real applications and identify functional faults [17], [18]. For the static test, a write operation is performed with a known data pattern (i,e. solid ' 0 ', solid ' 1 ' and checkerboard patterns), then the memory is irradiated during a time interval, and subsequently, a readback operation is performed to identify the corrupted bits.

For dynamic tests, four different algorithms were used: March C-, Dynamic Stress, Dynamic Classic, and mMats+ [19], [20]. Theses algorithms were previously used, as example, on SRAM [21], FRAM [22], MRAM [23], to evaluate the radiation impact on the devices. Fig. 3 to 6 depict the four algorithms, in which, the arrow indicates the addressing order (' $\uparrow$ ' up or ' $\downarrow$ ' down), ' $w$ ' (write) and ' $r$ ' (read) indicates the operation and the following Boolean number indicates 


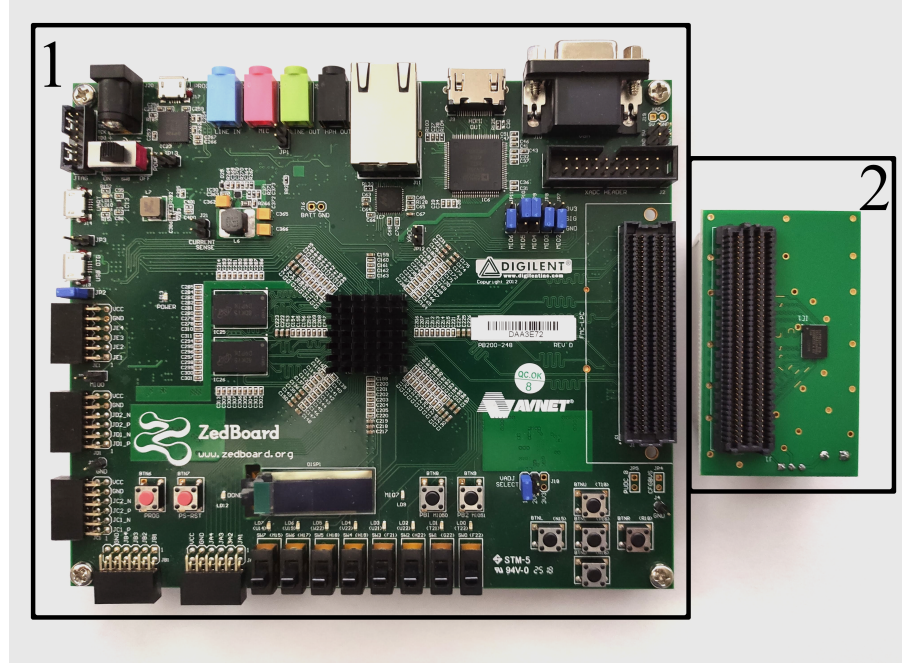

Fig. 2. Top-down photograph of the controller board (square number 1) and the daughter board (square number 2).

the data background. The algorithms are composed of elements which are indicated by the arrow followed by the operations within the parenthesis, each element is applied to the entire address space before proceeding to the following one, and a complete dynamic test algorithm is delimited by a bracket pair [24]. For the March C-, Dynamic Stress and mMats+, the first element (up write operation) is performed only once for the initialization of the memory.

$$
\begin{aligned}
& \uparrow(w 0) ; \\
& \{\uparrow(r 0, w 1) ; \uparrow(r 1, w 0) ; \downarrow(r 0, w 1) ; \downarrow(r 1, w 0) ; \uparrow(r 0)\}
\end{aligned}
$$

Fig. 3. Scheme of dynamic March C- test algorithm.

$$
\begin{aligned}
& \uparrow(w 1) ; \\
\{\uparrow & (r 1, w 0, r 0, r 0, r 0, r 0, r 0) ; \\
& \uparrow(r 0, w 1, r 1, r 1, r 1, r 1, r 1) ; \\
& \uparrow(r 1, w 0, r 0, r 0, r 0, r 0, r 0) ; \\
& \downarrow(r 0, w 1, r 1, r 1, r 1, r 1, r 1) ; \\
& \downarrow(r 1, w 0, r 0, r 0, r 0, r 0, r 0) ; \\
& \uparrow(r 0, w 1, r 1, r 1, r 1, r 1, r 1)\}
\end{aligned}
$$

Fig. 4. Scheme of Dynamic Stress test algorithm.

$$
\{\uparrow(w 0) ; \uparrow(r 0) ; \uparrow(w 1) ; \downarrow(r 1)\}
$$

Fig. 5. Scheme of Dynamic Classic test algorithm.

\section{REsults}

Static and dynamic tests were applied at the DUT in several runs of five minutes each, in order to have a fluence of $3 \times 10^{11}$ particles $/ \mathrm{cm}^{2}$.

The analysis of the test logs led to the identification of four different failures types. The simplest observed failure mode consists of SBUs (Single-Bit Upsets), which was observed 18 times. A write operation was sufficient to erase these SBUs, and the occurrence was not recurrent at the same bit address.

The phenomenon of stuck bits was observed in two different manners: permanent and temporary stuck bits. The failure is defined as a bit with a stuck value (' 0 ' or ' 1 ') independently of the value that was written. In this study, permanent stuck bits are the ones that, after the first appearance, the error occurs in each one of the following 


$$
\begin{aligned}
& \uparrow(w 0) ; \\
& \{\uparrow(r 0, w 1) ; \uparrow(r 1, w 0)\}
\end{aligned}
$$

Fig. 6. Scheme of dynamic mMats+ test algorithm.

read accesses to the faulty address. In the case of temporary stuck bits, the error returns just during a certain time window.

The number of permanent stuck bits as a function of cumulative fluence is presented in Fig. 7, which exhibit a growth of stuck cells with the increase of the cumulative fluence. The points depicted in this figure represents the number of stuck bits at their first appearance. During a Dynamic Stress test, all the cells that present the stuck at phenomenon do not return the faulty bit as an error in the sequential five read back performed just after a write operation, however, it appeared in the first read operation performed in the next element of the algorithm. This behavior can be explained with an induced reduction (by the particle interaction) of the retention time of the storage capacitor of the cell. The logic value of the stuck cell was either ' 0 ' or ' 1 ', showing that each logic value is represented by a charged or discharged capacitor depending on the memory region. From the total observed stuck (permanent and temporary) cells, $44.1 \%$ was stuck at ' 0 ' and $55.9 \%$ at ' 1 '.

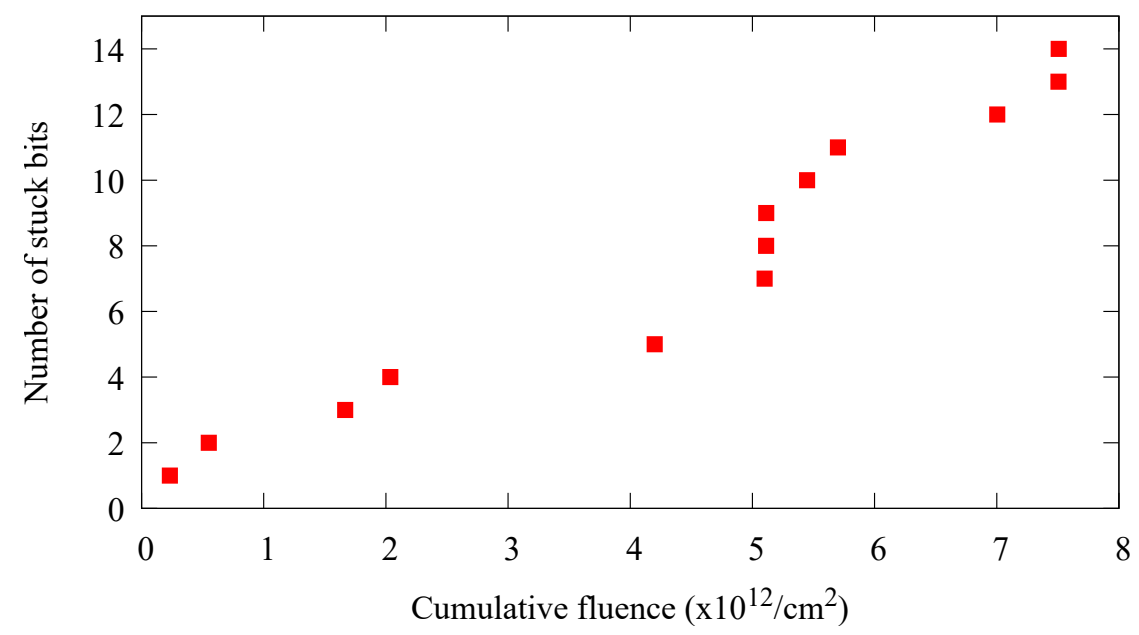

Fig. 7. Number of stuck bits in the DUT as a function of cumulative fluence.

Temporary stuck bits presented the same behavior of the permanent ones. The only difference is that, in the first case, the failure is not permanent and just occurred during consecutive write and read operations that were performed within the dynamic and static test modes. Temporary stuck bits also presented different level of damage, i.e. different retention capability of the cell. The duration of these temporary errors was different depending on the test run.

Besides the described failures, block errors with vertical and horizontal shapes were observed in the memory bitmaps. In order to evaluate these events, we generated logical bitmaps by dividing the memory array into two parts, using the left side for pair rows and the right side for the even ones. This procedure generated 16384 columns. In a bitmap, each pixel represents a bit cell.

An example of a horizontal block error can be seen in Fig. 8, which is the resulted Bitmap of a static test with a checkerboard pattern as data background. In the figure, two square zones are zoomed-in to increase visibility. These events are characterized by errors occurring in all the 512-word addresses of two consecutive even or odd rows, being most of the bits within a word with an error. An exception of this behavior is presented in the left zoomed-in square of Fig. 8, were within the same address range, the bitmap shows a horizontal band of errors with most of bits not faulty, resulting in events with less than the expected 1024 words errors.

Block errors were also observed with a vertical shape, in which the same column is affected in subsequent even or odd rows. Fig. 9 spot this block error identified during a Dynamic Stress test in a second cycle for the first " 11 " operation of the fourth element of the algorithm. It is interesting to highlight that in all vertical lines of errors, the addresses with errors span in the same range, returning a maximum of 2048 words with errors. 


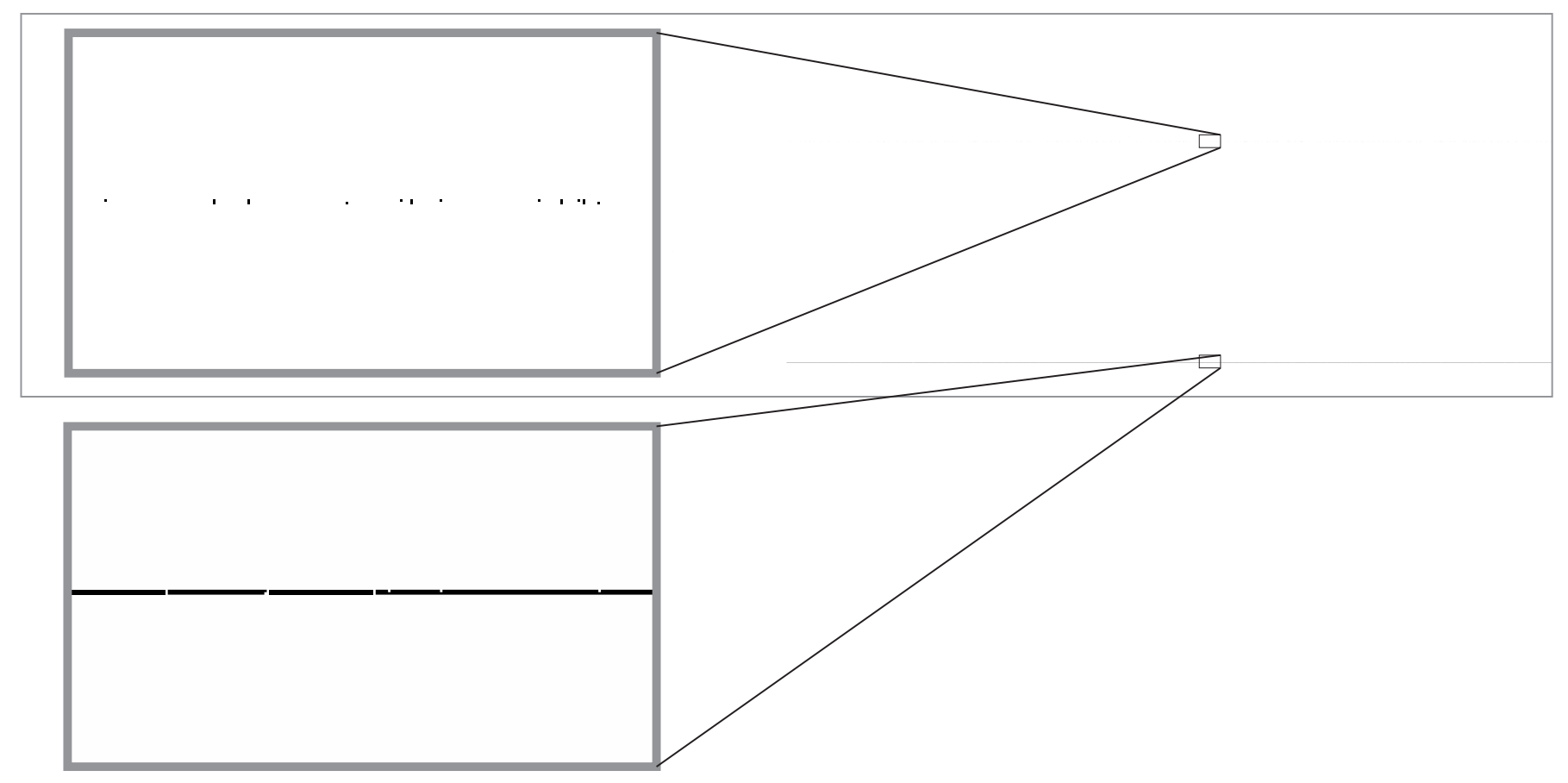

Fig. 8. Bitmap obtained after a static test mode using a checkerboard "AAAAh". Each pixel represents a bit; bits that were identified with errors appears in black. The gray lines are used to limit the region. Zoom-ins are added to increase the visibility of the horizontal block events.

For both vertical and horizontal errors, a write operation was able to restore the access to the cells without the need to carry out a power cycle. This type of errors is not due to problem related to the affected cells, but rather to the control logic. In particular, a temporary malfunction of the sense amplifier or register that serve that column may lead to this type of behavior.

Besides the above-discussed events, two blocks of errors spanning a different range of addresses occurred during the test campaign. The first event is depicted in Fig. 10. The Bitmap presented in the figure was obtained during a Dynamic Stress test. The red arrows show the six error lines that were presented in the five " 1 " operations performed in the last element of the Dynamic Stress algorithm. In this case, in three fixed columns in both even and odd rows, we identified twelve addresses range. As the opposite of the first vertical event, all the addresses returned all bits with an error.

The second type of vertical line failure mechanism was observed during March C- test execution, with increasing addressing order, resulting in a sequence of more than 100 words with errors. The affected addresses were dependent of the execution order, resulting in a range from "000000h" to "00006Ah" for an increasing order ( $\uparrow$ ), and from "3FFFFFh" down to "3FFF8Dh" for a decreasing order $(\downarrow)$. The effect persisted during several cycles of dynamic tests. However, after a dynamic execution, we performed static write and read operation, and the block error was recovered after two static writes, returning its appearance during the next dynamic test. This event occurred during five runs using March C-, Dynamic Classic, mMats+, and with a sequence of static tests between the irradiation runs, and it was totally recovered just after a power cycle.

In order to evaluate the events' cross-sections of this memory, the failure types were divided into SBUs, temporary stuck bits, permanent stuck bits, and block errors. The estimate event cross section $(\sigma)$ is defined as

$$
\sigma=\frac{N}{F \times M}
$$

where $N$ is the number of events, $F$ is the beam fluence in particles $/ \mathrm{cm}^{2}$, and $M$ is the number of bits 25.

From the calculated events cross sections, we define the SER, which expressed in FIT/Mb. FIT/Mb is equal to a failure per billions of working hours per $\mathrm{Mb}$ [26], [27]. The equation is

$$
S E R=\sigma \times(1024 \times 1024) \times 10^{9} \times j
$$

where $1024 \times 1024$ (bits) is the Mb coefficient, $10^{9}$ is the FIT definition, and $j\left(6.5\right.$ particles $\left./ \mathrm{cm}^{2} / \mathrm{h}\right)$ is the thermal energies' ( $<400 \mathrm{meV}$ ) flux at New York (sea level) outdoors for a mean solar activity defined in JEDEC JESD89B [1], [27], [28]. 


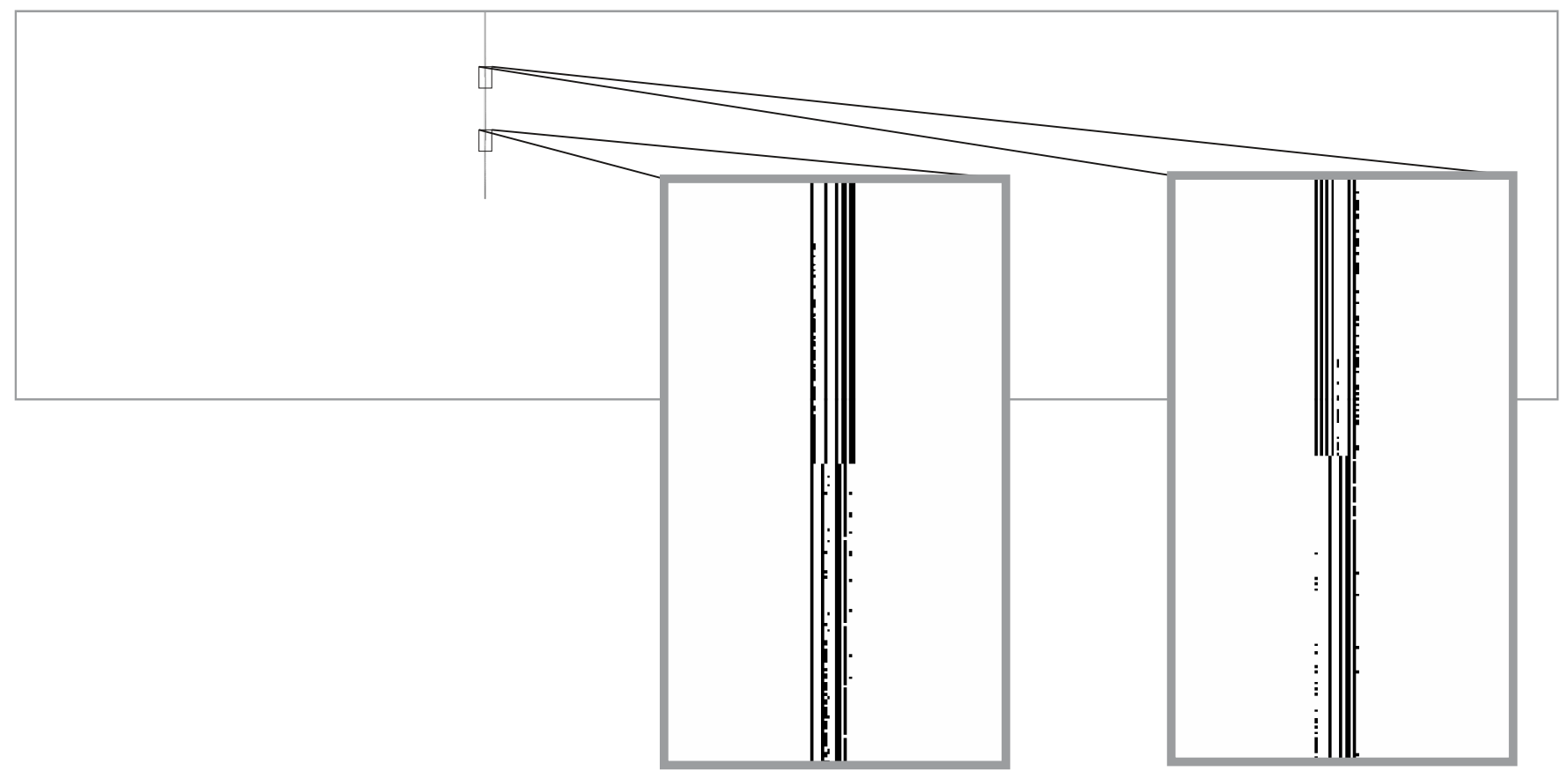

Fig. 9. Bitmap obtained during a Dynamic Stress test after the first ' $r 1$ ' of the fourth line of the algorithm. Each pixel represents a bit; bits that were identified with errors appear in black. The gray lines are used to limit the region. Zoom-ins are added to increase the visibility of the horizontal block events.

Table [1 presents the estimated cross sections and SER.

TABLE I

ESTIMATED CROSS SECTION AND SER FOR THE FOUR FAILURE TYPES IDENTIFIED IN THIS STUDY. THE VALUES WERE CALCULATED USING THE EQ. 1 AND 2

\begin{tabular}{ccc}
\hline Failure type & Cross Section $\left.(\sigma) \mathbf{~ c m}^{2} / \mathbf{b i t}\right)$ & SER (FIT/Mb) \\
\hline Single bit upset & $3.43 \times 10^{-20}$ & $2.3 \times 10^{-4}$ \\
Permanent stuck bit & $2.67 \times 10^{-20}$ & $1.7 \times 10^{-4}$ \\
Temporary stuck bit & $4.01 \times 10^{-20}$ & $2.7 \times 10^{-4}$ \\
Block errors & $2.67 \times 10^{-20}$ & $1.7 \times 10^{-4}$ \\
\hline
\end{tabular}

\section{CONCLUSION}

The effects of thermal neutron irradiation in a self-refresh DRAM were described. From static and dynamic test modes realized during a test campaign, different kinds of failures were identified. Besides the occurrence of SBUs, the tests showed permanent and temporary stuck bits, which already had been reported in several studies, presenting different fault mechanisms, being the most probable cause the irradiation impact on the variable retention time phenomenon [29], [30].

Furthermore, block errors were observed in four different patterns, with intermittent word errors in vertical and horizontal sequential logical addresses, and also presenting divided vertical lines with all bit within a word with errors, and a sequential error with dependency in the addressing order.

Cross-section for the different kinds of failures were estimated in a magnitude order of $10^{-20}$, showing that the memory is not very sensitive to thermal neutrons, however, it is necessary to consider that vertical and horizontal errors present a significant quantity of word errors within an event, where, for a user point of view, is relevant in critical applications.

\section{REFERENCES}

[1] C. Weulersse, S. Houssany, N. Guibbaud, J. Segura-Ruiz, J. Beaucour, F. Miller, and M. Mazurek, "Contribution of Thermal Neutrons to Soft Error Rate," IEEE Transactions on Nuclear Science, vol. 65, no. 8, pp. 1851-1857, aug 2018. 


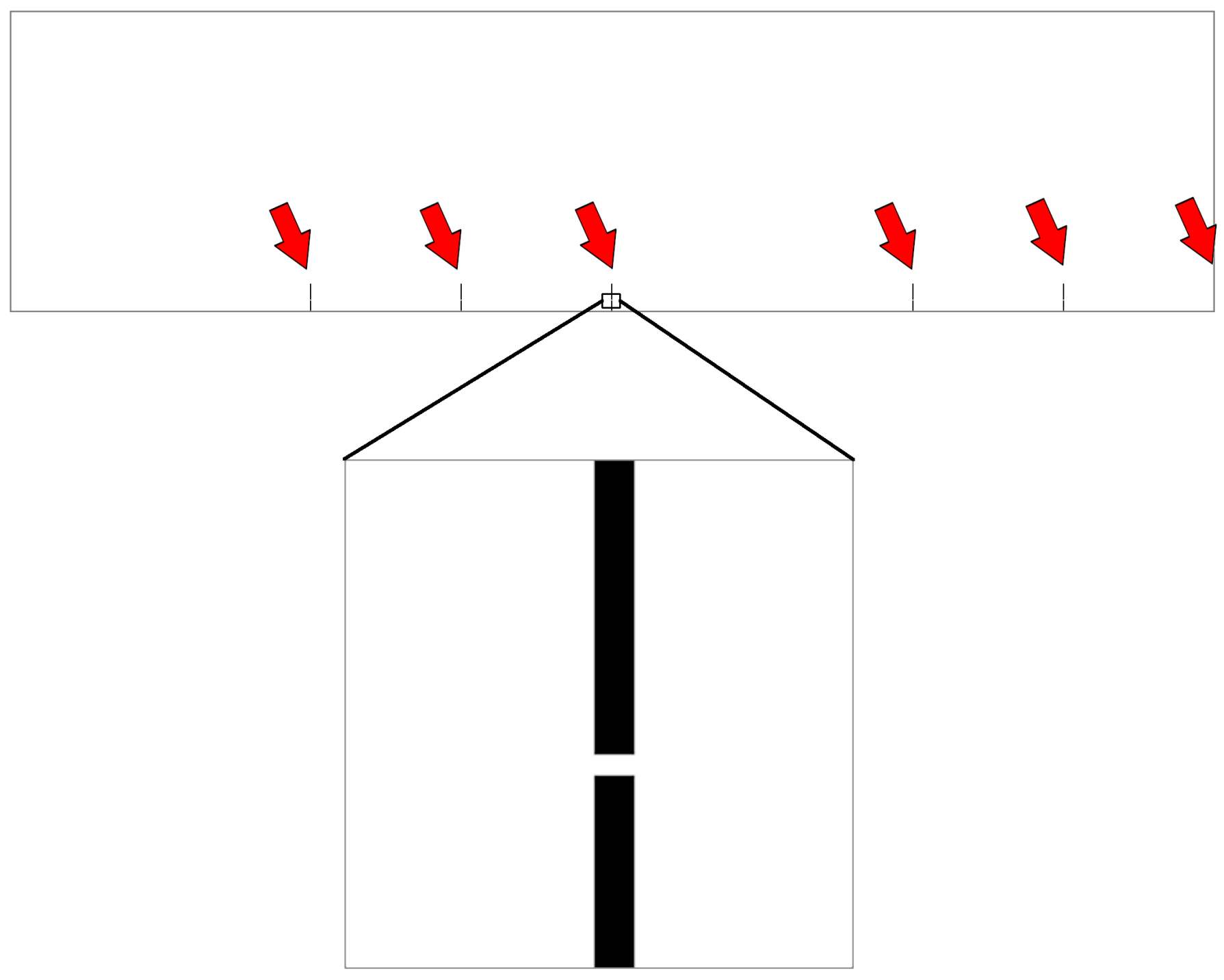

Fig. 10. Bitmap obtained during a Dynamic Stress test after the fifth 'ro' of the sixth line of the algorithm. Each pixel represents a bit; bits that were identified with errors appear in black. The gray lines are used to limit the region. Zoom-ins are added to increase the visibility of the horizontal block events. Red arrows indicate the six vertical lines.

[2] R. D. Baumann, "Section III-Landmarks terrestrial single-event effects," in Proc. IEEE Nucl. Space Radiat. Effects Conf. Short Course Notebook, San Francisco, CA. USA, 2013

[3] R. Baumann, T. Hossain, E. Smith, S. Murata, and H. Kitagawa, "Boron as a primary source of radiation in high density drams," in Digest of Technical Papers - Symposium on VLSI Technology. IEEE, 1995, pp. 81-82.

[4] E. H. Ibe, Terrestrial Radiation Effects in ULSI Devices and Electronic Systems. New York, New York, USA: Wiley-IEEE Press, 2014.

[5] S. J. Wen, R. Wong, M. Romain, and N. Tam, "Thermal neutron soft error rate for SRAMs in the $90 \mathrm{~nm}-45 \mathrm{~nm}$ technology range," in IEEE International Reliability Physics Symposium Proceedings, 2010, pp. 1036-1039.

[6] S. J. Wen, S. Y. Pai, R. Wong, M. Romain, and N. Tam, "B10 finding and correlation to thermal neutron soft error rate sensitivity for SRAMs in the sub-micron technology," in IEEE International Integrated Reliability Workshop Final Report, 2010, pp. 31-33.

[7] J. L. Autran, S. Serre, S. Semikh, D. Munteanu, G. Gasiot, and P. Roche, "Soft-error rate induced by thermal and low energy neutrons in $40 \mathrm{~nm}$ SRAMs," IEEE Transactions on Nuclear Science, vol. 59, no. 6, pp. 2658-2665, 2012.

[8] T. Yamazaki, T. Kato, T. Uemura, H. Matsuyama, Y. Tada, K. Yamazaki, T. Soeda, T. Miyajima, and Y. Kataoka, "Origin analysis of thermal neutron soft error rate at nanometer scale," Journal of Vacuum Science \& Technology B, Nanotechnology and Microelectronics: Materials, Processing, Measurement, and Phenomena, vol. 33, no. 2, mar 2015.

[9] Y.-P. Fang and A. S. Oates, "Thermal Neutron-Induced Soft Errors in Advanced Memory and Logic Devices," IEEE Transactions on Device and Materials Reliability, vol. 14, no. 1, pp. 583-586, mar 2014.

[10] M. Olmos, R. Gaillard, A. Van Overberghe, J. Beaucour, S. Wen, and C. Sung, "Investigation of thermal neutron induced soft error rates in commercial SRAMs with $0.35 \mu \mathrm{m}$ to $90 \mathrm{~nm}$ technologies," in IEEE International Reliability Physics Symposium Proceedings, 2006, pp. 212-216. 
[11] G. Tsiligiannis, S. Danzeca, R. Garcia-Alia, A. Infantino, A. Lesea, M. Brugger, A. Masi, S. Gilardoni, and F. Saigne, "Radiation Effects on Deep Submicrometer SRAM-Based FPGAs under the CERN Mixed-Field Radiation Environment," IEEE Transactions on Nuclear Science, vol. 65, no. 8, pp. 1511-1518, aug 2018.

[12] M. Cecchetto, R. Garcia Alia, S. Gerardin, M. Brugger, A. Infantino, and S. Danzeca, "Impact of Thermal and Intermediate Energy Neutrons on SRAM SEE Rates in the LHC Accelerator," IEEE Transactions on Nuclear Science, vol. 65, no. 8, pp. 1800-1806, aug 2018.

[13] P. Maillard, M. Hart, J. Barton, P. Jain, and J. Karp, "Neutron, $64 \mathrm{MeV}$ proton, thermal neutron and alpha single-event upset characterization of Xilinx 20nm UltraScale Kintex FPGA," in IEEE Radiation Effects Data Workshop, vol. 2015-November. Institute of Electrical and Electronics Engineers Inc., nov 2015.

[14] H. Zhang, H. Jiang, J. D. Brockman, T. R. Assis, X. Fan, B. L. Bhuva, B. Narasimham, S. J. Wen, and R. Wong, "Thermal neutron-induced soft-error rates for flip-flop designs in 16-nm bulk FinFET technology," in IEEE International Reliability Physics Symposium Proceedings. Institute of Electrical and Electronics Engineers Inc., may 2017, pp. 3D3.1-3D3.4.

[15] J. Beaucour, J. Segura-Ruiz, B. Giroud, E. Capria, E. Mitchell, C. Curfs, J. C. Royer, M. Baylac, F. Villa, and S. Rey, "Grenoble large scale facilities for advanced characterisation of microelectronics devices," in Proceedings of the European Conference on Radiation and its Effects on Components and Systems, RADECS, vol. 2015-December. Institute of Electrical and Electronics Engineers Inc., dec 2015.

[16] A. S. Kipcak, P. Gurses, E. M. Derun, N. Tugrul, and S. Piskin, "Characterization of boron carbide particles and its shielding behavior against neutron radiation," Energy Conversion and Management, vol. 72, pp. 39-44, 2013.

[17] A. Bosio, L. Dilillo, P. Girard, S. Pravossoudovitch, and A. Virazel, Advanced Test Methods for SRAMs. Boston, MA: Springer US, 2010.

[18] D. Niggemeyer, M. Redeker, and J. Otterstedt, "Integration of non-classical faults in standard March tests," in Proceedings - International Workshop on Memory Technology, Design and Testing, MTDT 1998, vol. 1998-August. Institute of Electrical and Electronics Engineers Inc., 1998, pp. 91-96.

[19] V. G. Mikitjuk, V. N. Yarmolik, and A. J. Van De Goor, "RAM testing algorithms for detection multiple linked faults," in Proceedings of the 1996 European Conference on Design and Test, EDTC 1996. Association for Computing Machinery, Inc, mar 1996, pp. 435-439.

[20] G. Tsiligiannis, L. Dilillo, V. Gupta, A. Bosio, P. Girard, A. Virazel, H. Puchner, A. Bosser, A. Javanainen, A. Virtanen, C. Frost, F. Wrobel, L. Dusseau, and F. Saigne, "Dynamic test methods for COTS SRAMs," IEEE Transactions on Nuclear Science, vol. 61, no. 6, pp. 3095-3102, dec 2014.

[21] P. Rech, J. M. Galliere, P. Girard, A. Griffoni, J. Boch, F. Wrobel, F. Saigne, and L. Dilillo, "Neutron-induced multiple bit upsets on two commercial SRAMs under dynamic-stress," IEEE Transactions on Nuclear Science, vol. 59, no. 4 PART 1, pp. 893-899, 2012.

[22] V. Gupta, A. Bosser, G. Tsiligiannis, A. Zadeh, A. Javanainen, A. Virtanen, H. Puchner, F. Saigne, F. Wrobel, and L. Dilillo, "Heavy-ion radiation impact on a 4Mb FRAM under different test conditions," in Proceedings of the European Conference on Radiation and its Effects on Components and Systems, RADECS, vol. 2015-December. Institute of Electrical and Electronics Engineers Inc., dec 2015.

[23] G. Tsiligiannis, L. Dilillo, A. Bosio, P. Girard, A. Todri, A. Virazel, S. S. McClure, A. D. Touboul, F. Wrobel, and F. Saigne, "Testing a commercial MRAM under neutron and alpha radiation indynamic mode," IEEE Transactions on Nuclear Science, vol. 60, no. 4, pp. 2617-2622, 2013.

[24] S. Hamdioui, Testing Static Random Access Memories, ser. Frontiers in Electronic Testing. Boston, MA: Springer US, 2004, vol. 26.

[25] E. Petersen, Single Event Effects in Aerospace. John Wiley \& Sons, 2011.

[26] C. Leroy and P.-G. Rancoita, Principles of Radiation Interaction in Matter and Detection, 4th ed. World Scientific, feb 2016.

[27] G. Tsiligiannis, E. I. Vatajelu, L. Dilillo, A. Bosio, P. Girard, S. Pravossoudovitch, A. Todri, A. Virazel, F. Wrobel, and F. Saigne, "SRAM soft error rate evaluation under atmospheric neutron radiation and PVT variations," in Proceedings of the 2013 IEEE 19th International On-Line Testing Symposium, IOLTS 2013, 2013, pp. 145-150.

[28] JEDEC Standard 89B (JESD89B), "Measurement and Reporting of Alpha Particle and Terrestrial Cosmic Ray-Induced Soft Errors in Semiconductor Devices JESD89A JEDEC," 2006.

[29] A. M. Chugg, J. McIntosh, A. J. Burnell, P. H. Duncan, and J. Ward, "Probing the nature of intermittently stuck bits in dynamic RAM cells," in IEEE Transactions on Nuclear Science, vol. 57, no. 6, dec 2010, pp. 3190-3198.

[30] V. Goiffon, A. Jay, P. Paillet, T. Bilba, T. Deladerriere, G. Beaugendre, A. Le-Roch, A. Dion, C. Virmontois, J.-M. Belloir, and M. Gaillardin, "Radiation Induced Variable Retention Time in Dynamic Random Access Memories," IEEE Transactions on Nuclear Science, pp. 1-1, nov 2019. 